Dept. of Pharmacology and Toxicology, Fac. of Vet. Med., El Baath University, Hama, Syria.

\title{
EFFECT OF CICHORIUM INTYPUS ALCOHOLIC EXTRACT ON BLOOD GLUCOSE AND TOTAL CHOLESTEROL LEVELS IN DIABETIC RABBITS
}

(With 2 Tables and 2 Figures)

By

\section{SALWA EL DEBS; A.R. HAMOWIEH and M.A. SHALABY*}

* Dept. of Pharmacology, Fac. of Vet. Med., Cairo Univ., Giza, Egypt. (Received at 15/9/2011)

تأثير الخلاصة الكحولية لنبات الهندباء البري على مستوى السكر والكولسترول الكلي في دم الأرانب المصنابة بلداء البري السكري ملئوى البكري

سلوى الدبس ، عبل الرزاق حموية ، مصطفى عباس شلبي

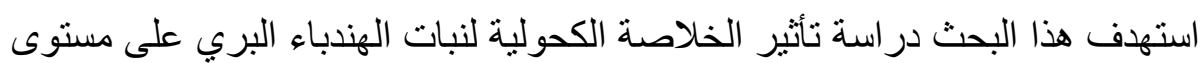

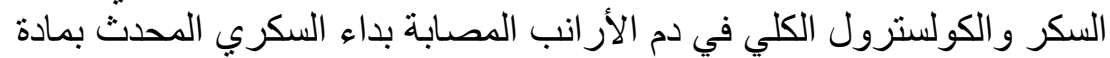

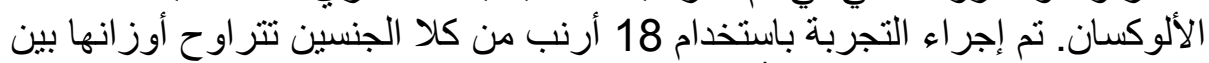

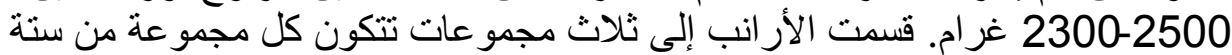

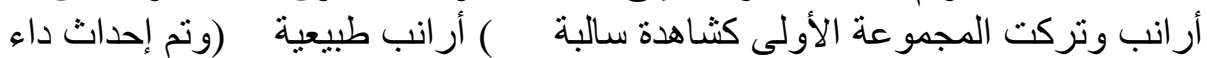

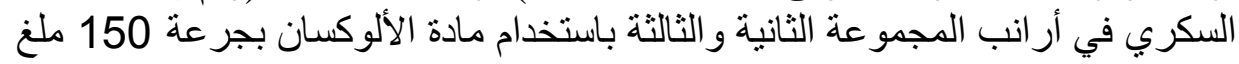

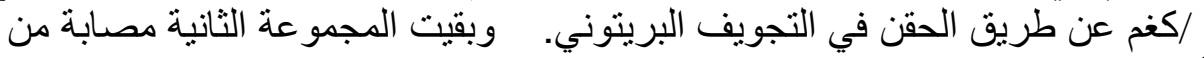

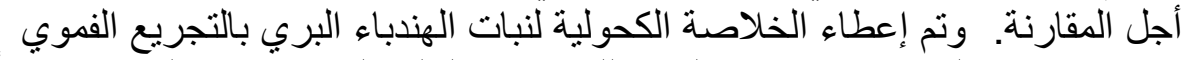

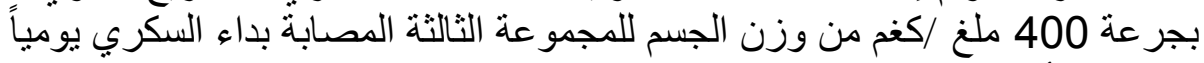

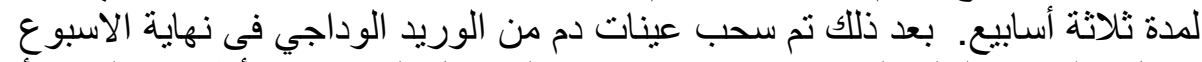

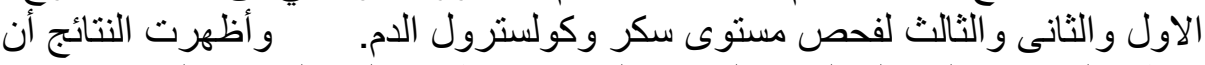

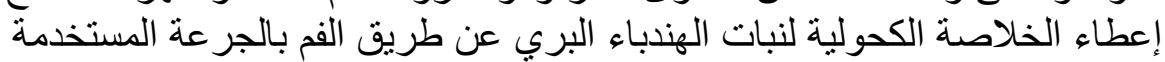

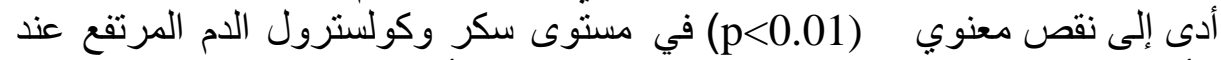

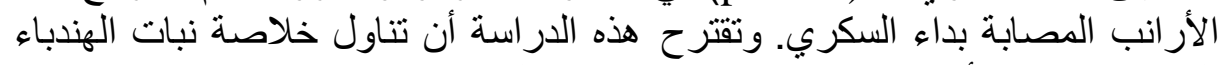

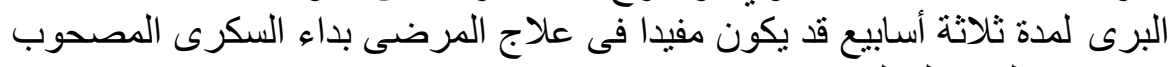
بارتفاع كولسترول الدام.

\section{SUMMARY}


The objective of this study was to investigate the effect of alcohol extract of Cichorium Intybus on blood glucose and total cholesterol levels in alloxan- diabetic rabbits. The experiment was carried out on 18 rabbits of both sexes and body weight ranged between 2300$2500 \mathrm{~g}$ b.wt. Rabbits were divided into 3 groups of 6 animals each. The $1^{\text {st }}$ group was kept as a normal control, while rabbits of the $2^{\text {nd }}$ and $3^{\text {rd }}$ groups were rendered diabetic by intraperitoneal injection of alloxan in a dose of $150 \mathrm{mg} / \mathrm{kg}$ b.wt. The $2^{\text {nd }}$ group was left as a diabetic control, while rabbits of the $3^{\text {rd }}$ group were orally given the alcohol extract of Cichorium Intybus in a dose of $400 \mathrm{mg} / \mathrm{kg}$ b.wt./ day for 3 weeks. Blood samples were collected at end of the $1^{\text {st }}, 2^{\text {nd }}$ and $3^{\text {rd }}$ week for determination of glucose and total cholesterol levels. The obtained results showed that oral administration of alcohol extract of Cichorium Intybus significantly decreased the high blood glucose and toloal cholesterol levels of the treated diabetic rabbits, as compared to the diabetic control group. In conclusion Oral administration of Cichorium Intybus alcohol extract produces antidiabetic and hypocholesterolemic effects in alloxan-diabetic rabbits. Intake of Cichorium Intybus for 3 weeks may be beneficial for patients who suffer from diabetes mellitus accampanied with hypercholesterolemia.

Key words: Cichorium intypus, blood glucose, total cholesterol, diabetic rabbits

\section{INTRODUCTION}

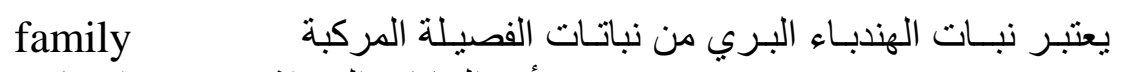
Composite

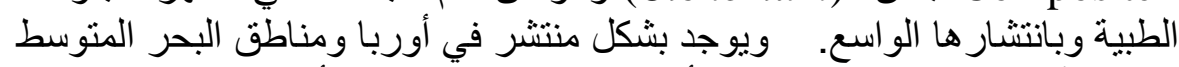

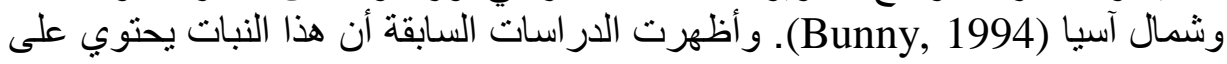

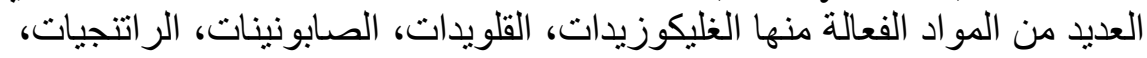

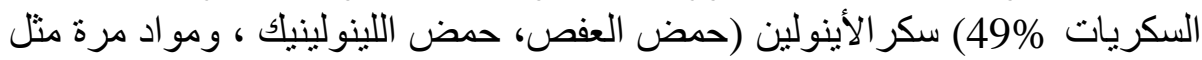

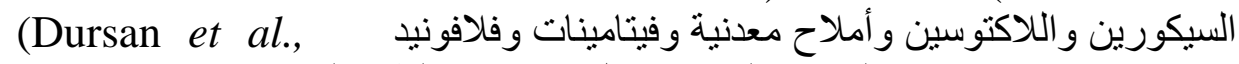

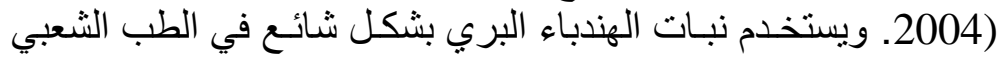

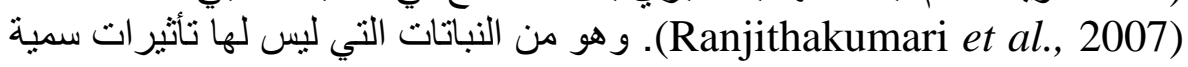


Akhtar et al. ويدراسة سمية النبات ذكر الباحث البناء (Pushparaj et al., 2007)

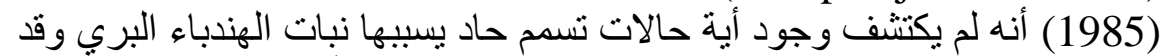

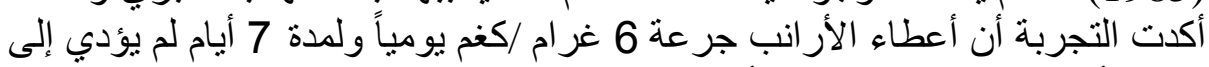
ظهور أية علامات تسم على الأرانب.

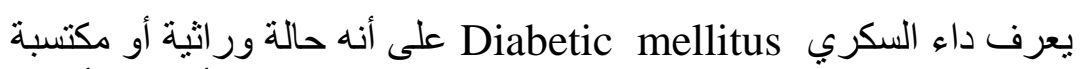

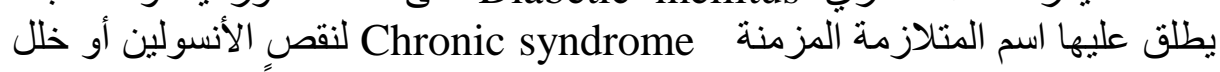

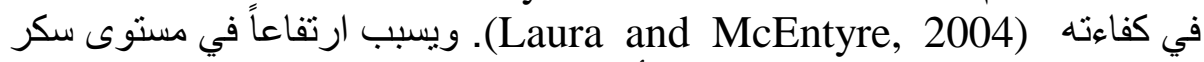
الدم (Yin et al., 2004). واستخدم الألوكسان كمادة كيميائية بجر عة الأنة 150 ملغ /كغم

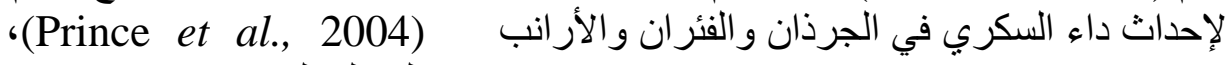
(Billis et al., 2002)

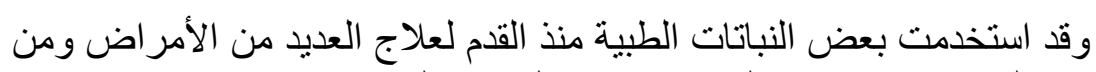

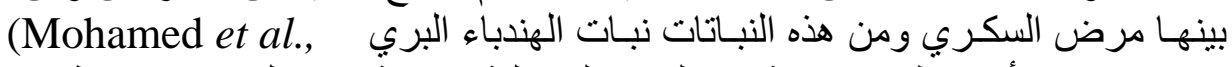

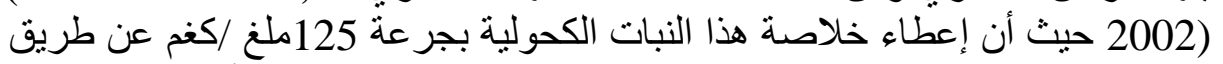

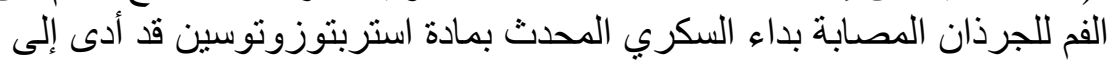

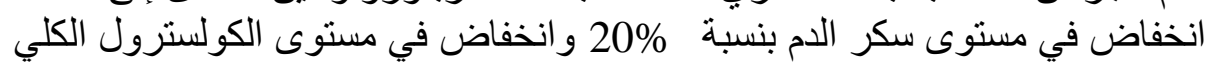
بنسبة 16\% و انخفاض في مستوى الثحوم الثناثية بنسبة Pushpara et al. (2007)

ووجد الباحث (1096) . Kok et al أن إضافة جذور نبات الهندباء إلى عليقة

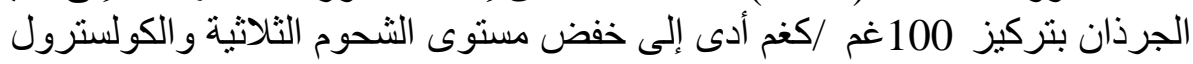

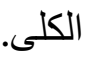

ولقد ذكر الباحث Hoon et al. أن نبات الهندباء البري يفيد في والي

علاج تلف خلايا الكبد والطحال و البنكرياس و الغذة التيموسية.

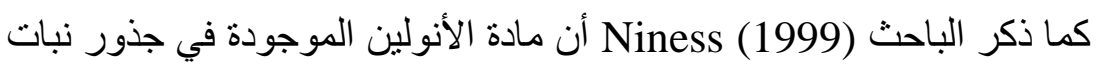

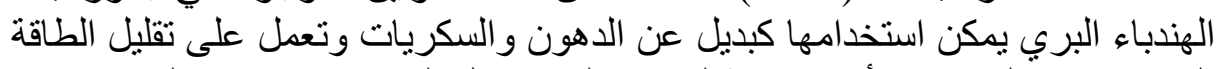

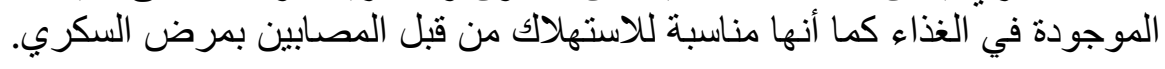

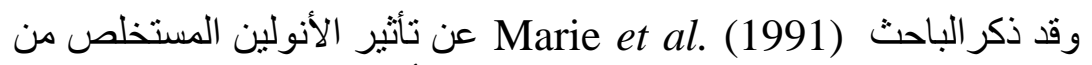

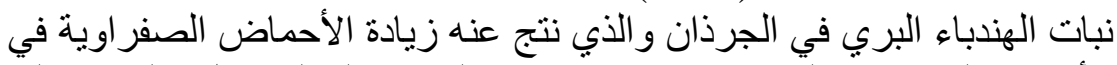

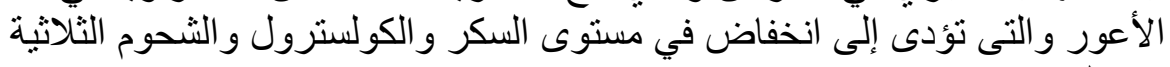

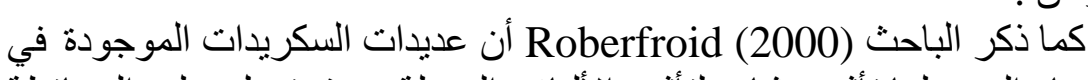
نبات الهندباء البري لها تأثيثر مشابه لتأثثر الألياف المنحلة حيث تعمل على الثى المحافظة

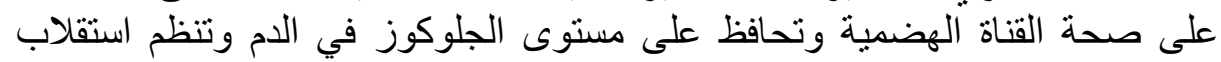

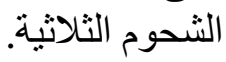




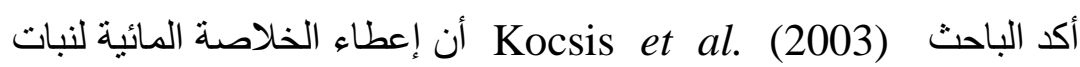

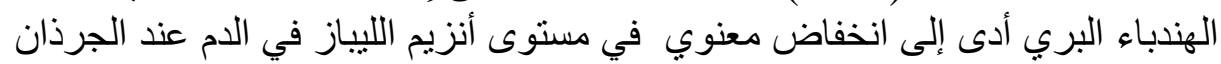
التي تعاني من فرط الثحوم التجريبي. أنغين

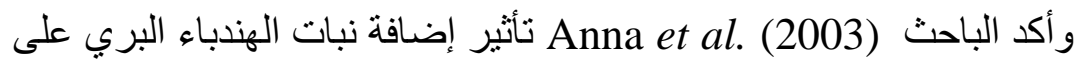

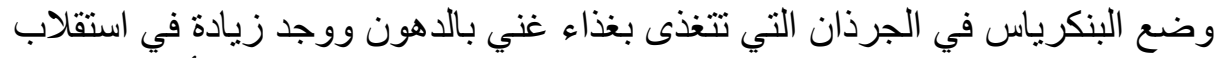

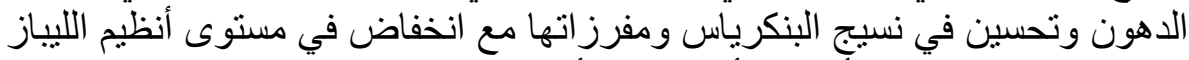

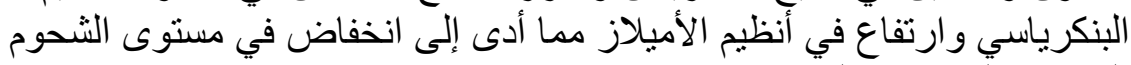
الثلاثية و السكر في الأنماع.

وقد ذكر (Urias et al. أن الأنولين المستخلص من نبات الهندباء البري ينظم الثهية وينظم استقلاب الثحوم و السكريات في الجسم.

وقد ذكر الباحث (1997) .Tiedge et al أن خلاصة نبات الهندباء تخفض

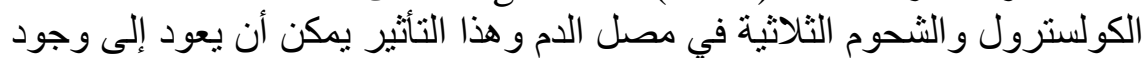

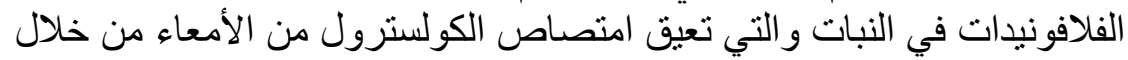

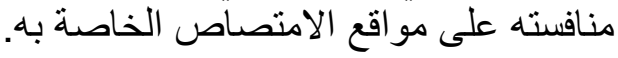

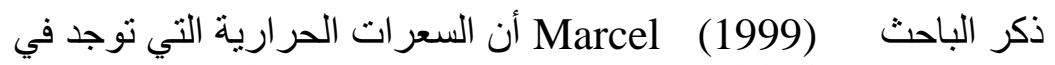

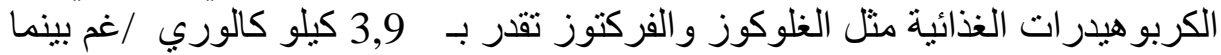

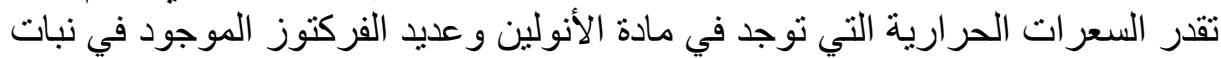

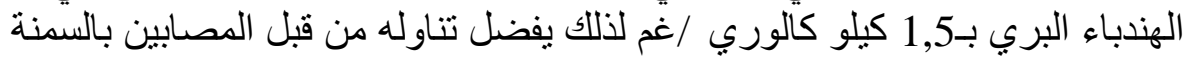
وداء السكري.

هدف البحث: استهدف هذا البحث دراسة نأثير الخلاصة الكحولية لنبات الهندباء البري

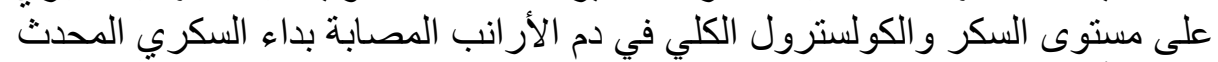

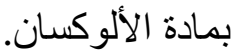

\section{MATERIALS and METHODS المواد وطرائق البحث}

أولاً: تحضير الخلاصة النباتية الكحولية:

تم الاعتماد على الطريقة الموصوفة فئة من فبل الباحثين

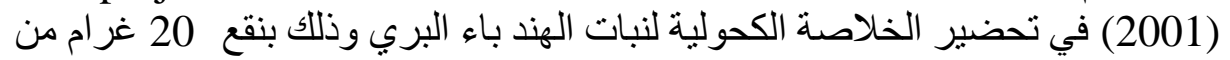

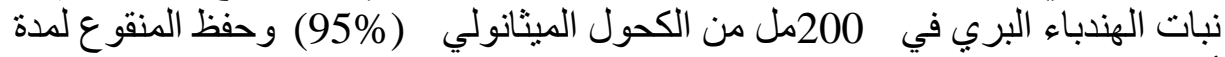

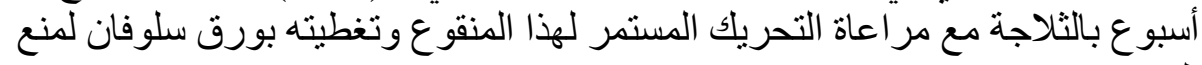
التبخر.

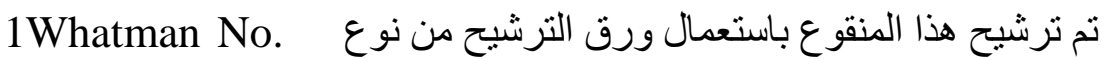
وتم عَرض الر اشح للطرد المركزي بقوة 2500 دورة /دقيقة لمدة 5 دقائق وتم التبخير 


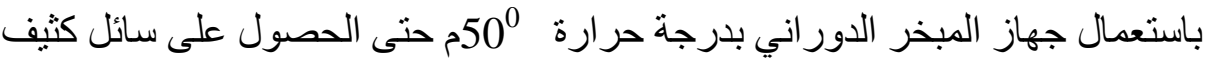

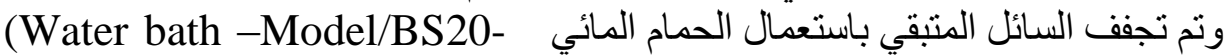
بamato-Japan)

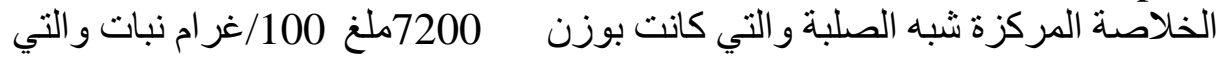

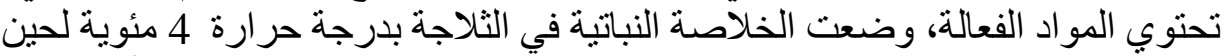

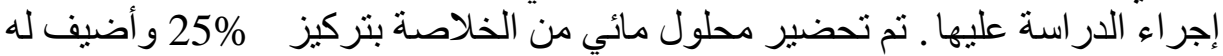
مادة tween80 بنسبة الاء 2\% محلول معلق.

ثانياً: حيوانات التجربة: 18 أرنب من كلا الجنسين في التجربة و تم شر اؤ ها من السوق

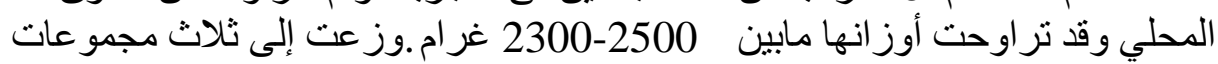

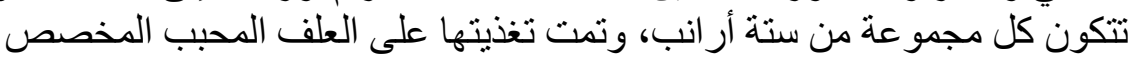
للأر انب وكان الماء متاح لهم بكمية كافية طيلة فترة التجربة.

ثالثاً: إحداث داء السكري بالألوكسان : تم تصويم أر انب المجمو عة الألوكئن الثانية و الثالثة عن الطعام لمدة 24 ساعة باستثناء

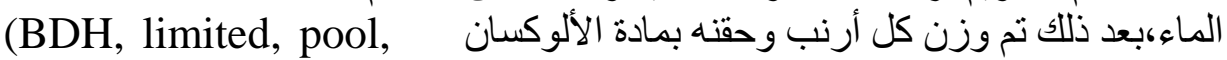

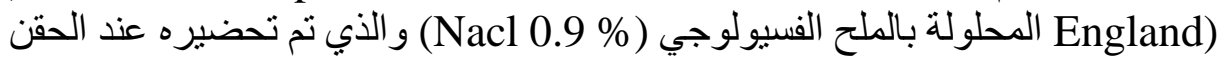

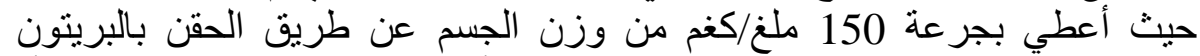

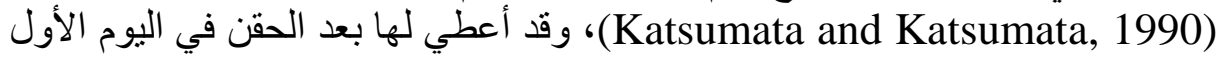

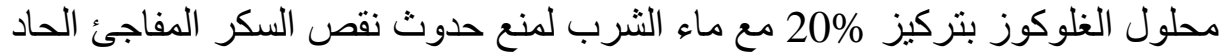

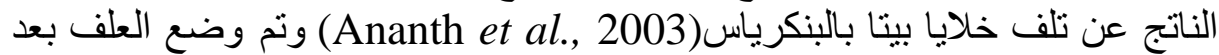

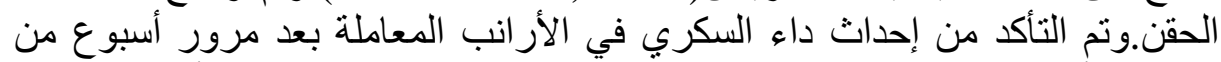

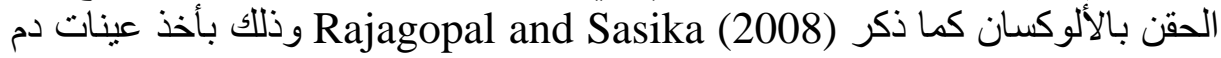

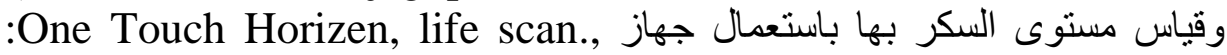
USA sensor)

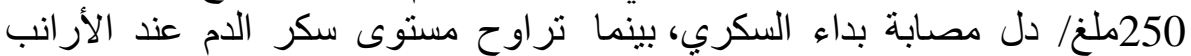

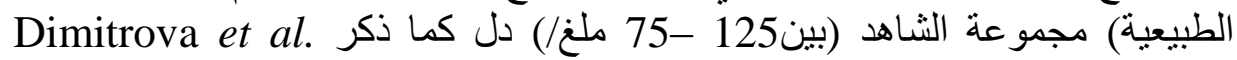
(2008)

رابعاً: التجربة: تم تقسيم مجمو عات التجربة على النحو التحو التالي : ـ المجموعة الأولى : مجمو عة شاهدة (أر انب طبيعية) ـ المجموعة الثانية : محقونة بالألوكسان: مصابة بداء السكري ومعاملة بالماء المقطر المضاف له 80 tween بنسبة 2\% حتى نهائة 
ـ المجموعة الثالثة محقونة بالألوكسان مصابة بداء السكري ومعاملة بالخلاصة

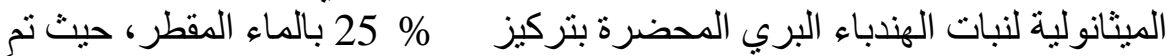

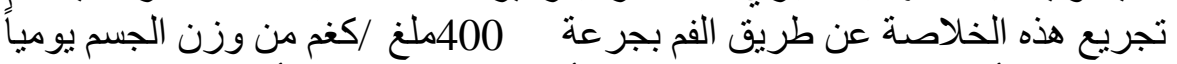

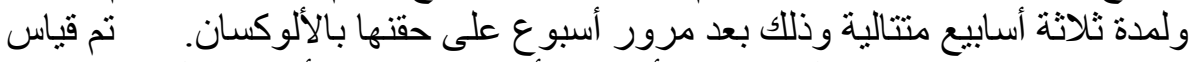

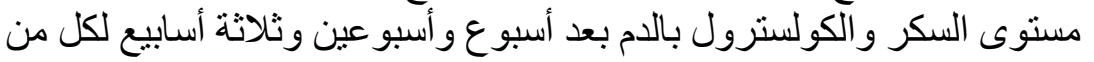

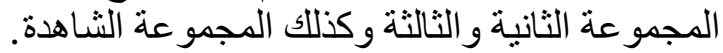

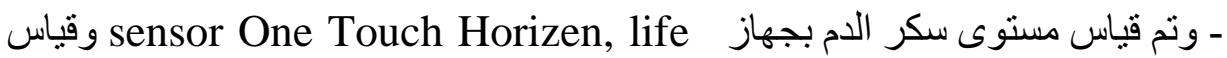
Richmond مستوى الكولسترول باستخدام الطريقة الكيميائية كما ذكر الباحث

One way ANOVA test استخدم اختبارتحليل التباين احادي الاتجاه

خامساً: التحليل الإحصائي:

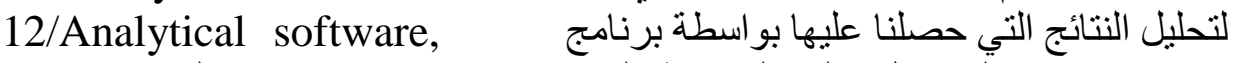
Version

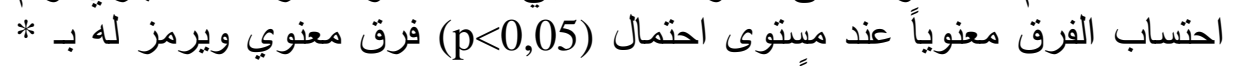

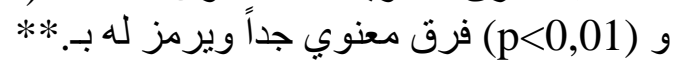

\section{RESULTS}

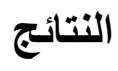

1 - 2 - مستوى سكر الام:

يبين جدول رقم ( 1) تركيز سكر الدم في (mg/dl) في المجمو عات الثناث للأر انب وتأثير

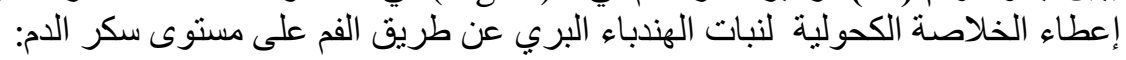


Assiut Vet. Med. J. Vol. 57 No. 131 October 2011

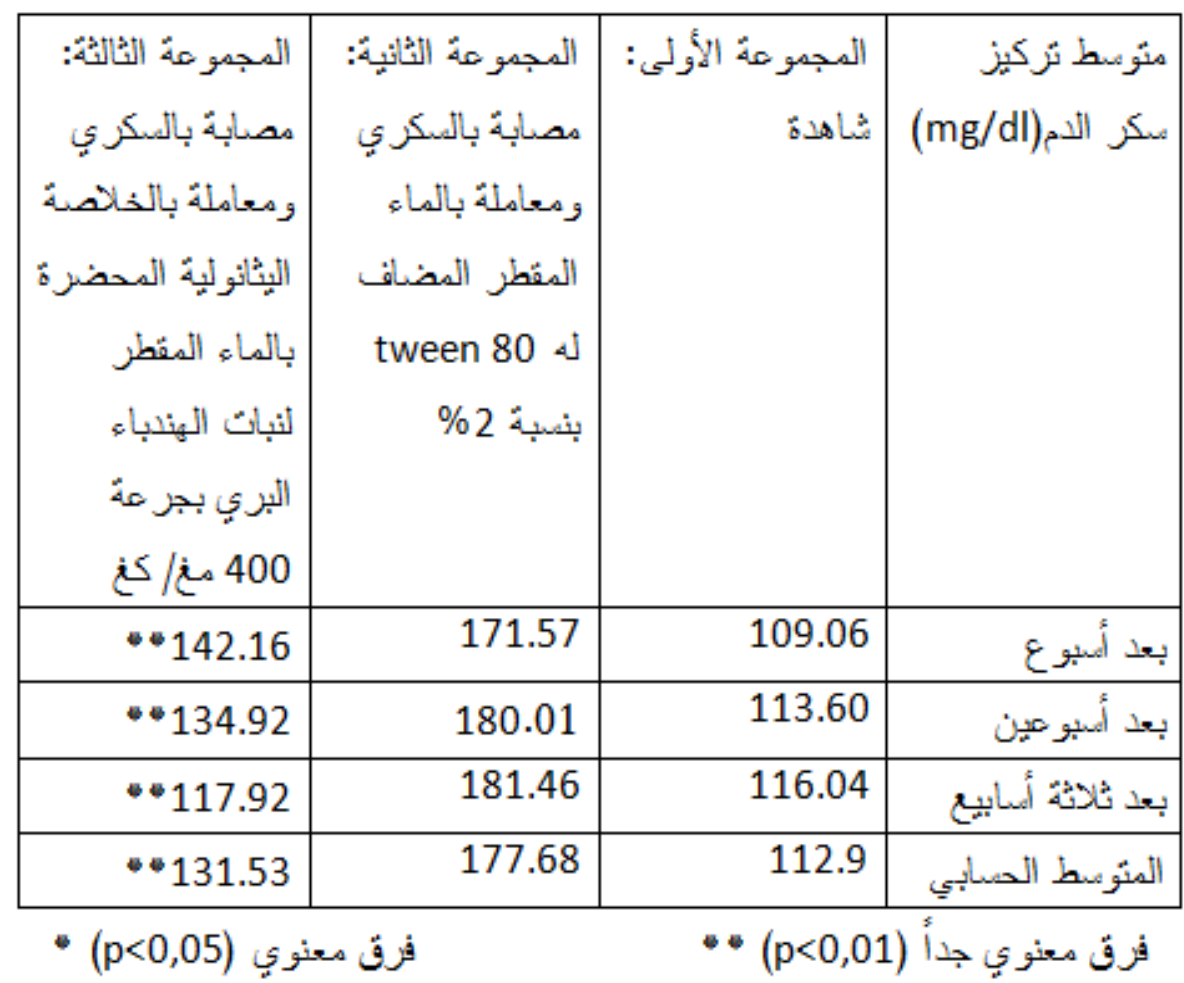

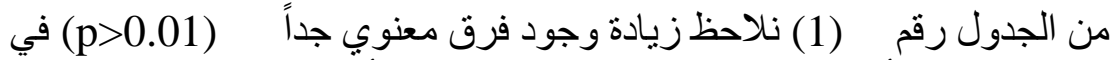

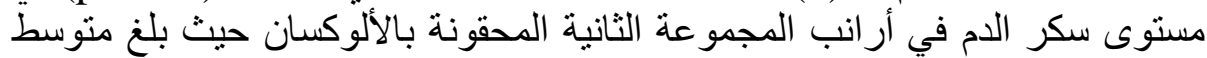

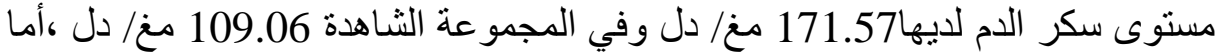

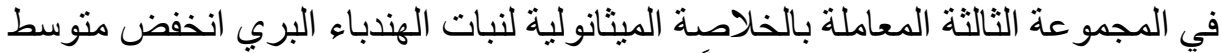

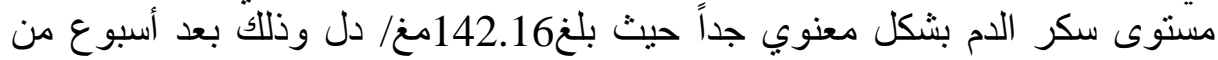
المعاملة بالخلاصة الكحولية.

أما بعد أسبو عين من المعاملة بالخلاصة الميثانولية لنبات الهندباء البري نلاحظ

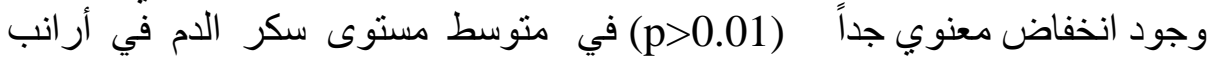

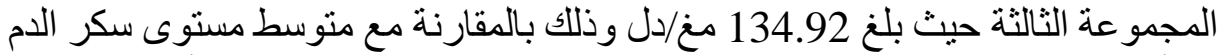

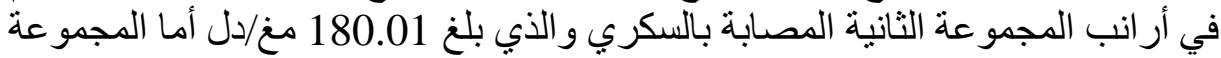
الثَاهدة فكان متوسط مستوى سكر الدم فيها. 113.60 وفي نهاية الأسبوع الثالث من المعاملة بالخلاصة الميثانولية لنبات الهندباء البري

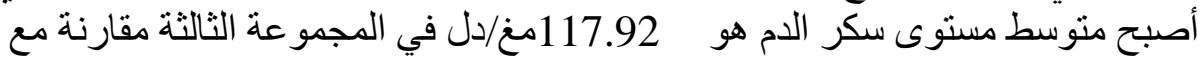
المجمو عة الثانية المصابة بالسكري التي كان متوسط مستوى سكر الدم عندها 181.64مغ/دل. 
المخطط البياني رقم 1 : يبين تأثير المعاملة بالخلاصة الكحولية لنبات الهندباء البري على مستوى سكر الدم/mg/dl في مجمو عات أر انب التجربة في الأسابيع الثناثة.

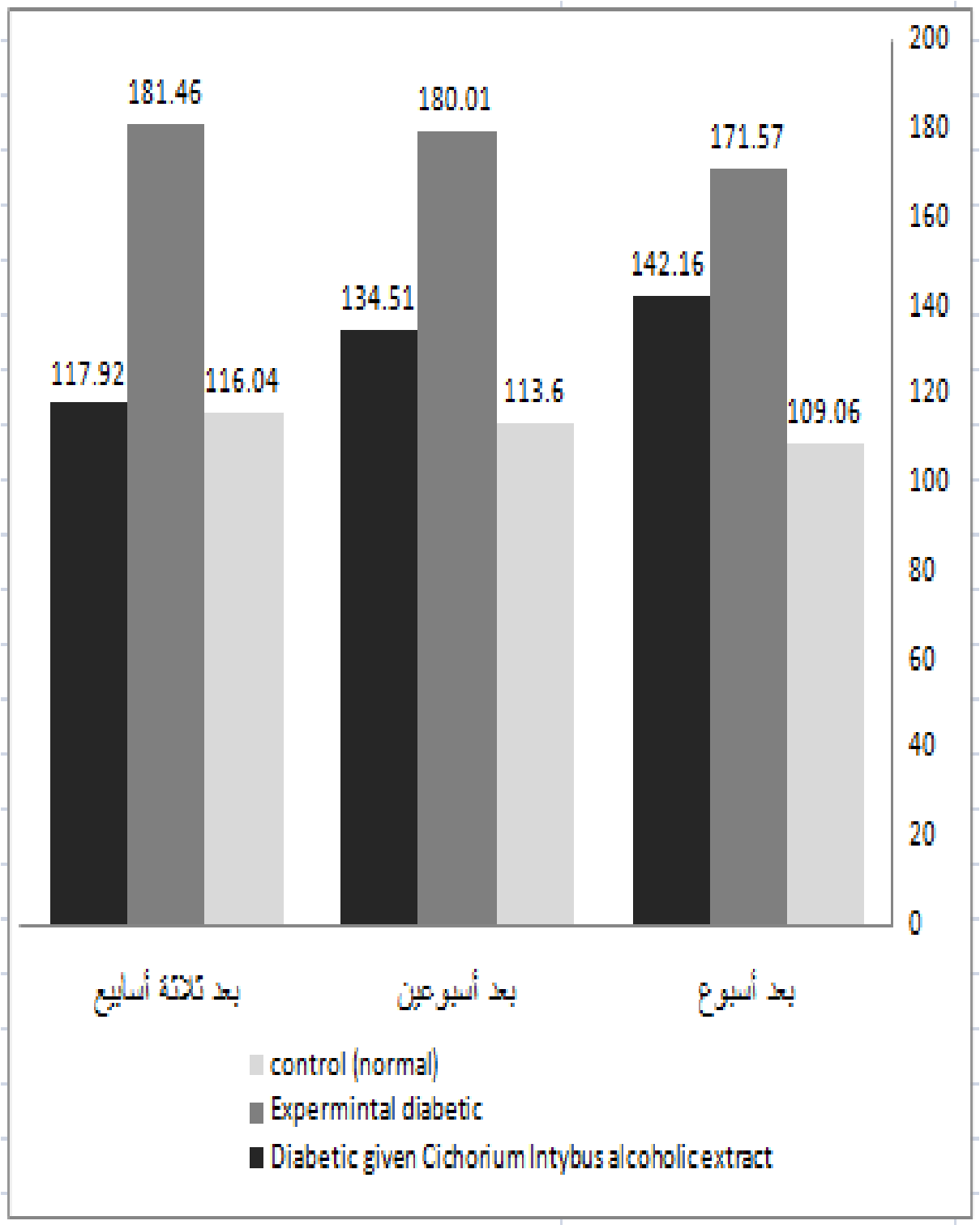


جلول رقم ( 2) يبين مستوى كولسترول الدم في إناء (mg/dl) في المجمو عات الثناث

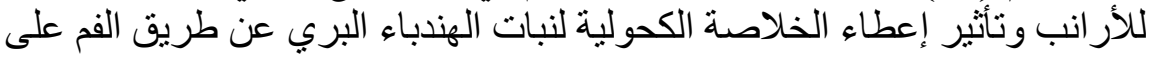

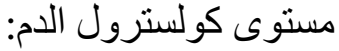

\begin{tabular}{|c|c|c|c|}
\hline المجمو عة الثالثة: & المجمو عة الثانية: & المجموعة الأولى: & منؤسط تركيز \\
\hline دصابة بالهكر بي & مصابة بالمكري & شاهدة & كولدشنرل \\
\hline ومعاملة بالخالضصة & ومعاملة بالماء & & الام(mg/dl) \\
\hline اليثانولية المحضرة & المقطر المضاف له & & \\
\hline بالماء المقطر لنبات & بئسبة tween 80 & & \\
\hline الهندباء البري & $\% 2$ & & \\
\hline بجر عة 400 هيغ/ & & & \\
\hline كغ & & & \\
\hline -39.67 & 45.26 & 36.50 & بعد أُسبوع \\
\hline 55.36 & 65.67 & 39.13 & بعد أُعبو عين \\
\hline 53.83 & 77.17 & 40.17 & بعد ثُلانثة أُمابيع \\
\hline$* 49.62$ & 62.7 & 38.60 & المئونسط الحصابي \\
\hline
\end{tabular}

نلاحظ من الجدول رقم (2) أن المعاملة بالخلاصة الميثانولية لنبات الهندباء

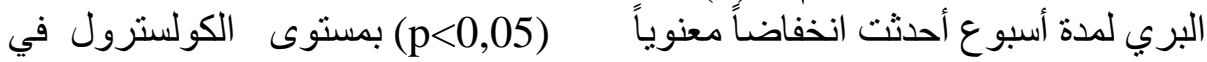

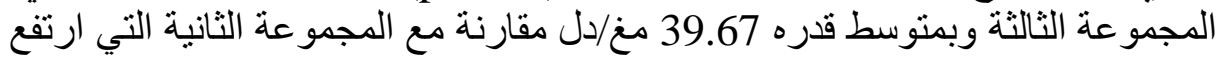

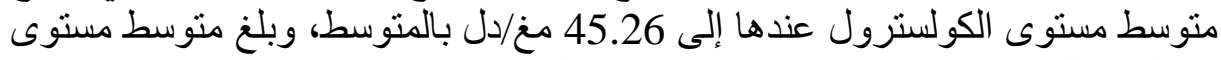

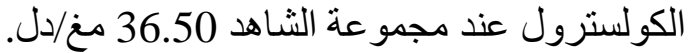

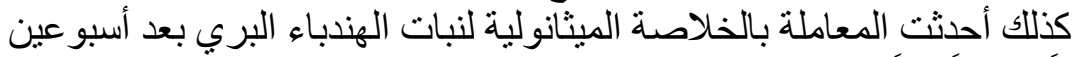

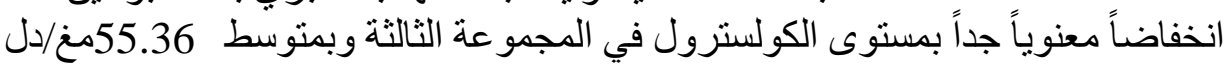

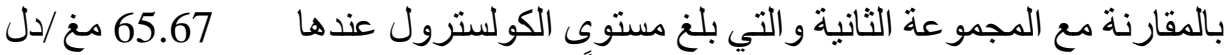

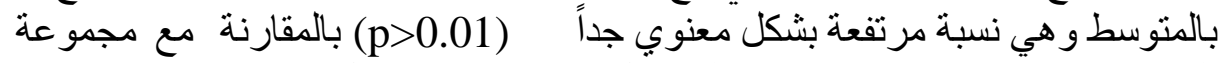
الثاهد التي بلغ متوسط مستوى الكولسترول فيها منسا 39.13 مغلدل. 
وفي نهاية الأسبوع الثالث من المعاملة بالخلاصة الميثانولية لنبات الهندباء البري

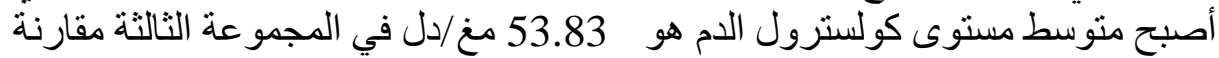

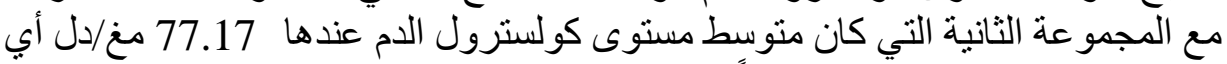
انخفض المنوسط بشكل معنوي جداً ( p (p)0.01)

المخطط البياني رقم ( 2): يبين تأثير المعاملة بالخلاصة الكحولية لنبات الهندباء البري على مستوى كولسترول الدم mg/dl في مجمو عات أر انب التجربة في الأسابيع

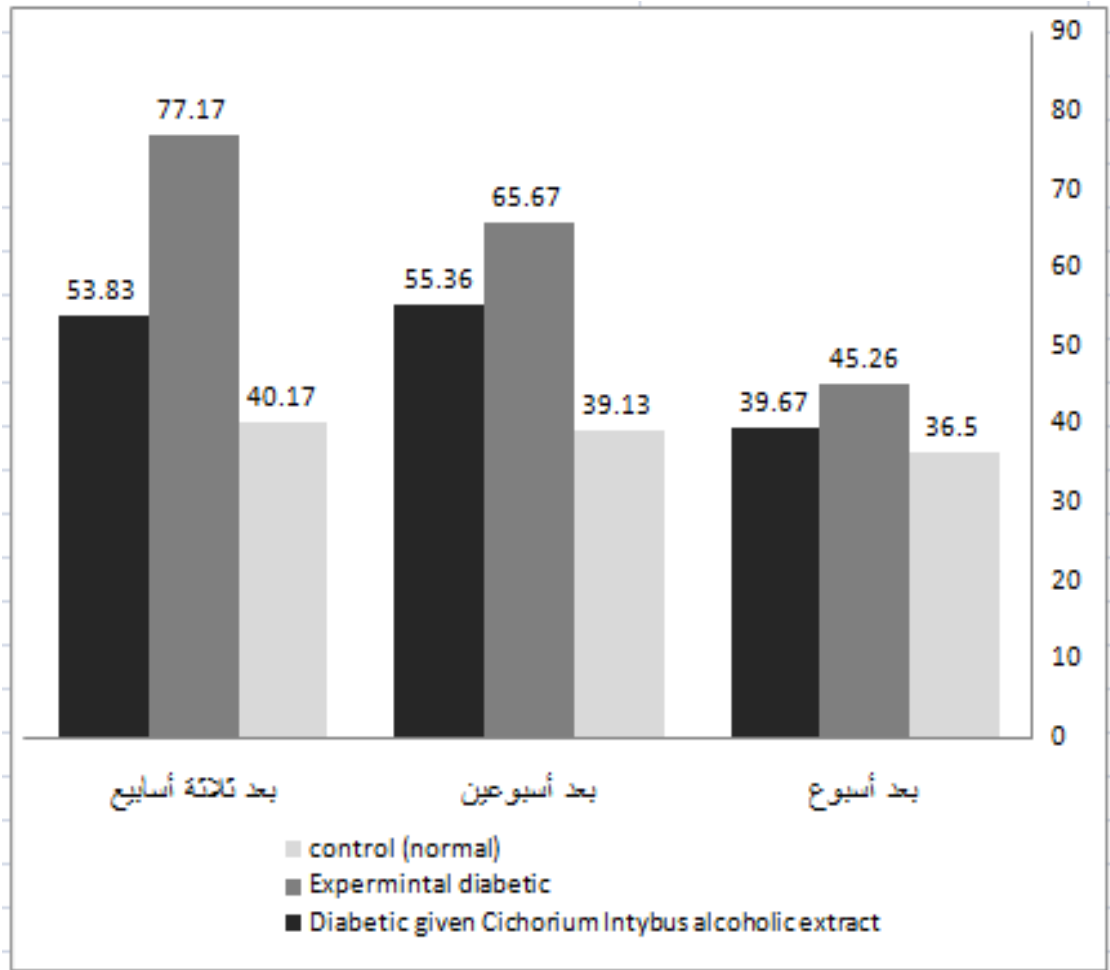

\section{DISCUSSION}

\section{المناقشـة}

أكد العالم Mc-Letchie (2002 فعالية الألوكسان في إصابة الأر انب بداء

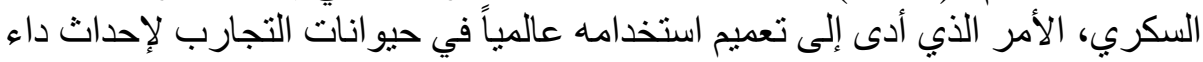

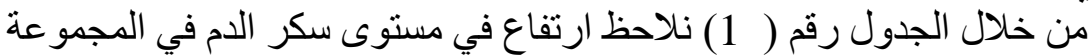
السكري.

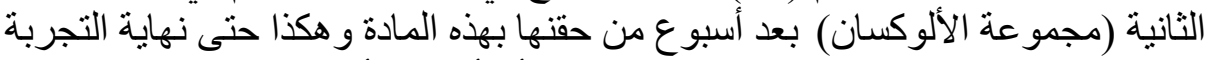

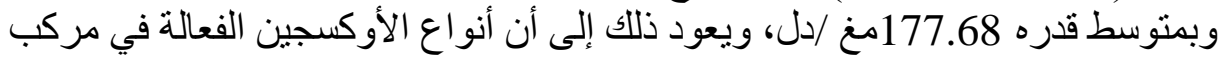


الألوكسان لها القدرة على مهاجمة جزر لانغر هانز في البنكرياس وبالتحديد خلايا بيتا

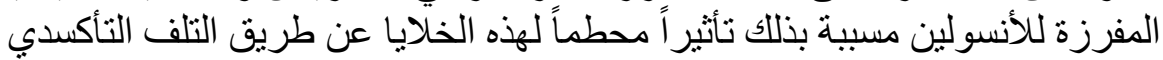

(Tiedge et al., 1997)

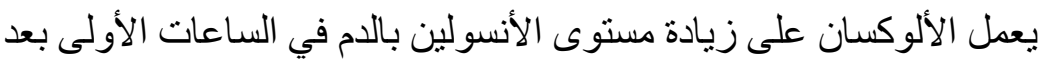

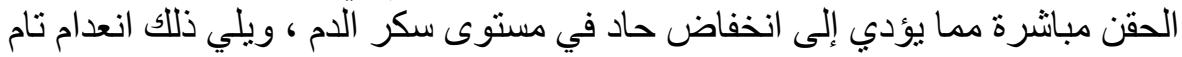

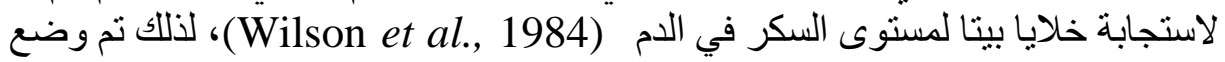
محلول سكري 20\% بماء الثرب لتفادي حدوث التياء الصدمة.

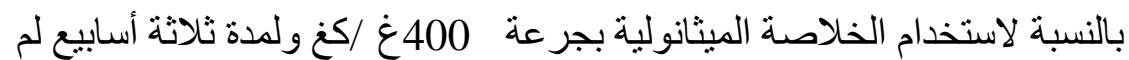

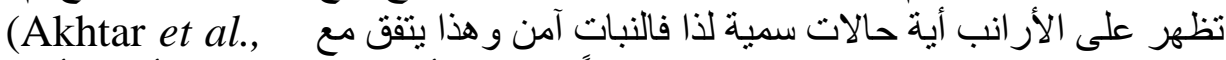

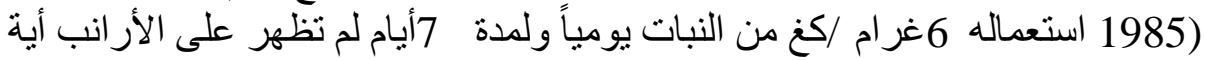
علامات للتسمم لذا فالنبات آمن باستعماله من النهات

و إن النقص الحاصل في مستوى سكر الدم في المجموعة الثالثة بألثة المعاملة

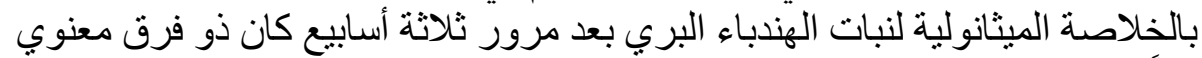

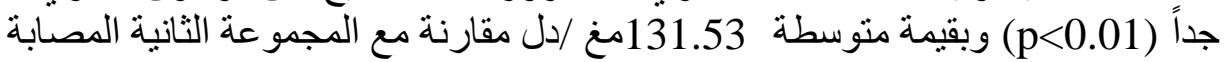

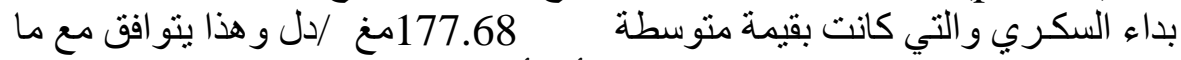

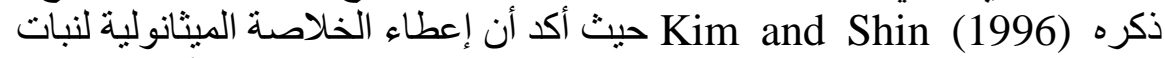
الهندباء البري للجرذان يؤدي إلى نقص في امتصاص الغلوكوز من الأمعاء الدقيقة

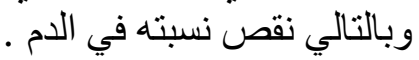

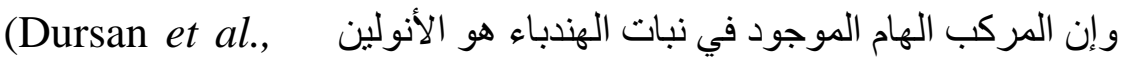

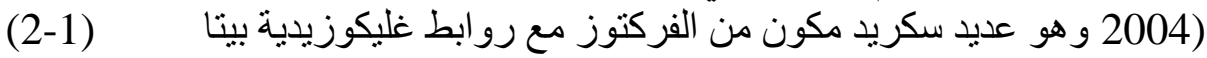

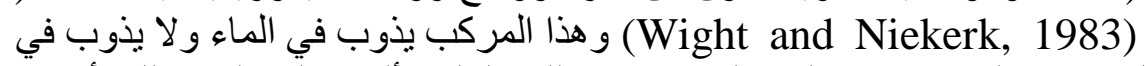
المفرزات الموجودة في القناة الهضمية ويسلك سلوك ولك الألياف المنحلة ويملك تأثنير

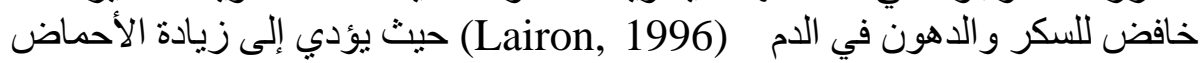

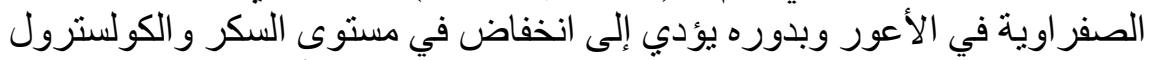

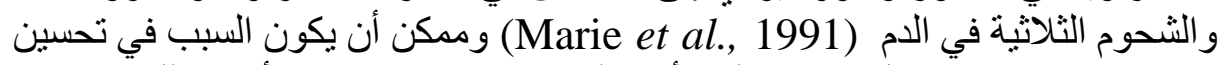

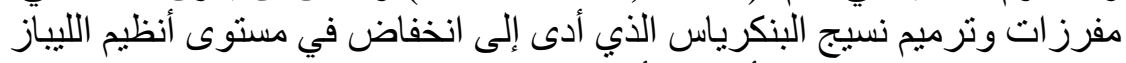

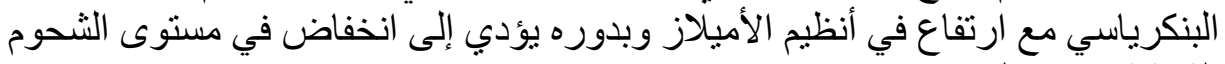

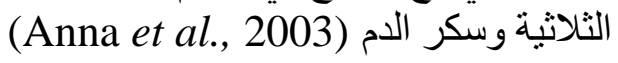

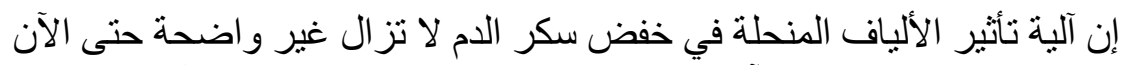

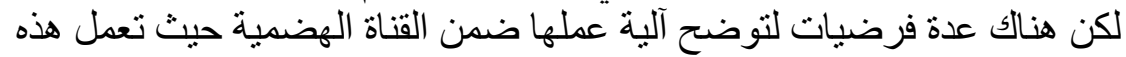

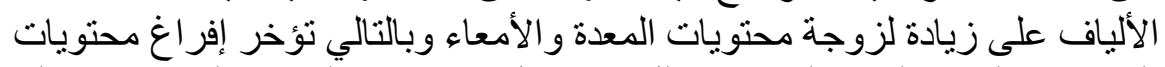

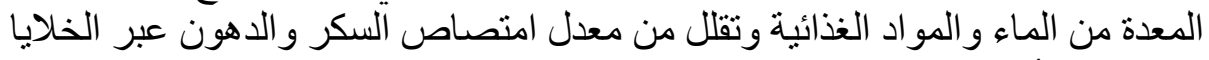

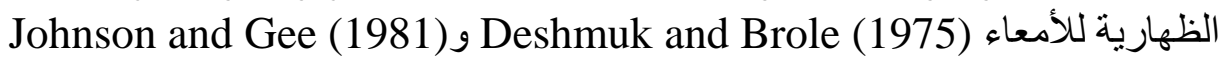

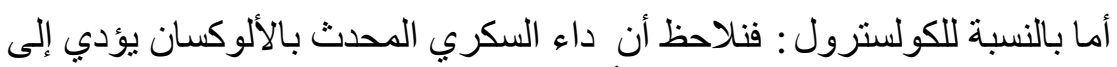

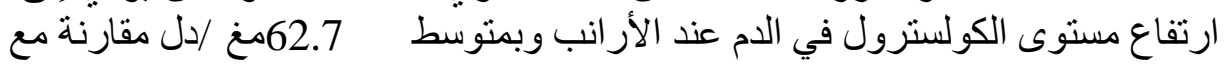


الثـاهد وبمتوسط قدره 38.60مغ /دل و هذا يتو افق مع النتائج التي حصل عليها كل من

Rao et al. (1999); Prince et al. (2004) إنى (2003) (1993); Sharma et al.hussary (19)

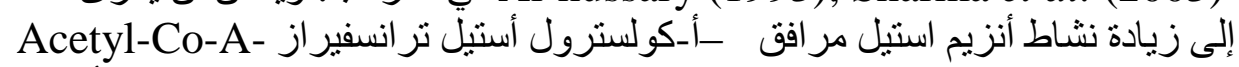

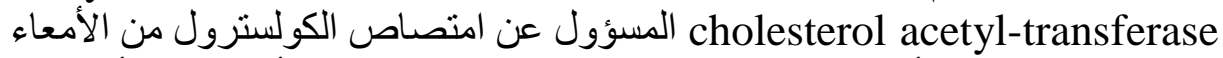
والتي تتحفز بغياب الأنسولين (Maechler et al., 1993) كما أن غياب الأنسولين الأنياء أدى إلى زيادة مستوى الكولسترول في الام (Lenich et al., 1990).

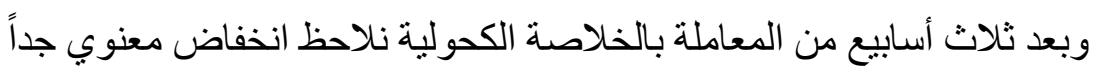

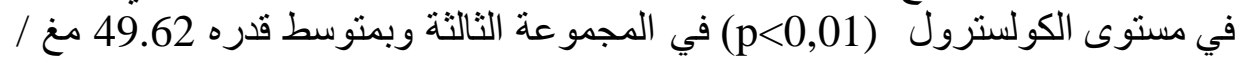

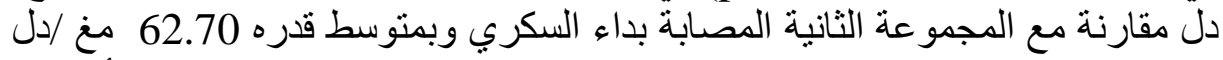
و هذا يتو افق مع ما ذكره (1996) .Kok et al ويعزى هذا الانخف النفاض إلى عدة أسباب:

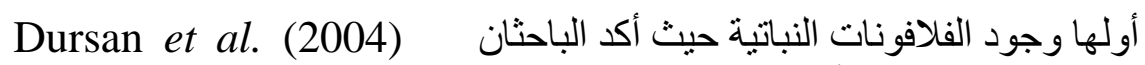

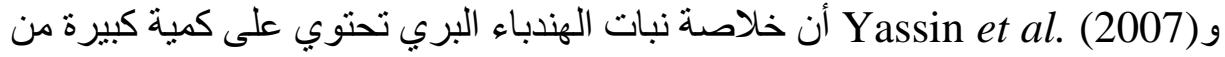
الفلافونات تصل إلى 58.1مغ/غرام .

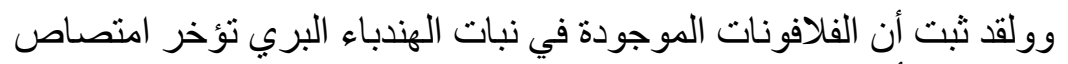

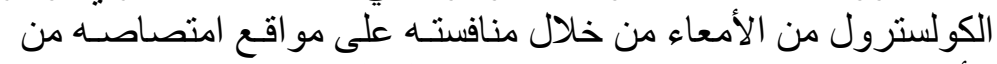

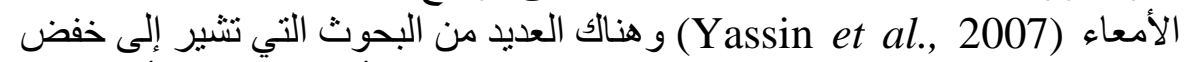

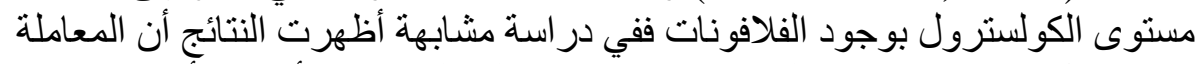

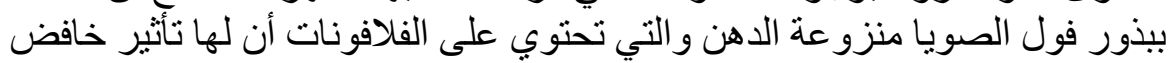

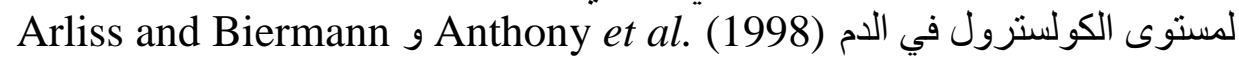
.(2002)

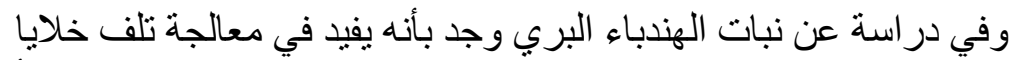

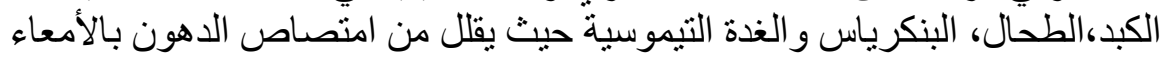

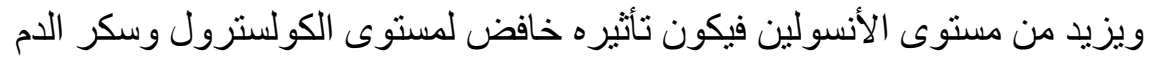
(Hoon et al., 2001)

وقد يعود هذا الانخفاض في مستوى الكولسترول لاحتو اء خلاصة نبات الهندباء

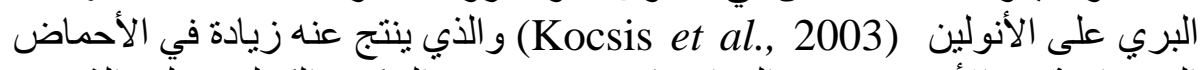

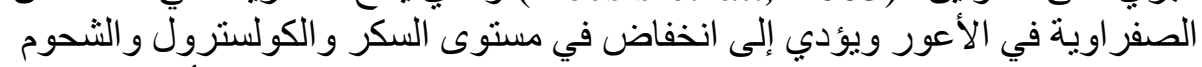

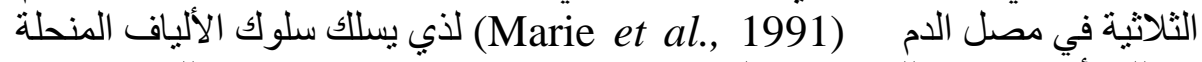
ويملك تأثير خافض للاهون في الدم (Kim and Shin, 1996) كذلك يؤدي نبات الأل الهندباء إلى زيادة استقلاب الدهون (Anna et al., 2003)

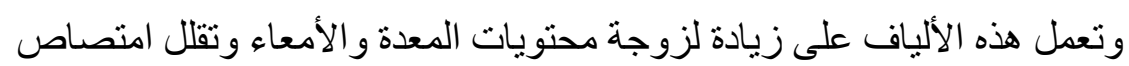

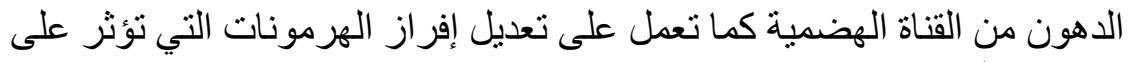
استقلاب الدهون (Johnson and Gee, 1981) 


\section{REFERENCES

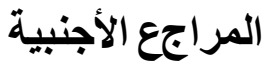

Akhtar, M.S.; Khan, Q.M. and Khaliq, T. (1985): Effect of Protulaca oleracae and Taraxacum officinale in normoglycaemic and alloxan- treated hyperglycemic rabbits. J. Pakstain Med. Assoc., 35: 207- 210.

Al-hussary, N. (1993): Effect of decoction of Fenugreek seeds on blood glucose, cholestrol and triglycerides in normal and alloxan diabetic rabbits.Iraqi J. Vet. Med., 1(6): 102-105.

Ananthan, R.; Latha, M.; Ramakumar, K.M.; Pari, L.; Baskar, C. and Narmatha, B. (2003): Effect of Gymnema montanum leaves on serum and tissue lipids in alloxan diabetic rats. Exp. Diabetes Res., 4(3): 183-189.

Anthony, M.S.; Clarkson, T.B. and Williams, J.T. (1998): Effects of isoflavones on atherosclerosis potential mechanisms. Am. J. Clin. Nutri., 68 (6): 1390S-1393S.

Arliss, R.M. and Biermann, C.A. (2002): Do Soy Isoflavones Lower Cholesterol, Inhibit atherosclerosis and play a role in cancer preventation. Pakstian J. Nutri., 16(5): 40-48.

Billis, L.S.; Shehu, R.A. and Abubakar, M.G. (2002): Hypoglycemic and hypolipidemic effects of aqueous extract of Arachis hypogaea in normal and alloxan diabetic rats. Phutomed., 6(9): 553-555.

Bunny, S. (1994): The illustrated Encyclopedia of herbs, their medicinal and culinary uses. Bunes and Nobles books, New York. USA. Page 246.

Deshmuk, S. and Brole, M. (1975): Studies on insecticidal properties of indigenous plant products. J. Ethropharmacol., 11: 18- 37.

Dimitrova, S.S.; Georgive, I.P.; Kanelov, I.N.; Iliev, Y.I. and Taner, S.I. (2008): Intravenous glucose tolerance test and glucose pharmacokinetic in rabbits. Bulg. J. Vet. Med., 11(3):161-169.

Dursan, E.; Semib, O. and Eren, A. (2004): Hebrs as food source in Turkey. Asian Pac. Can. Preven., 5: 334-339.

Johnson, I.M. and Gee, J.M. (1981): Effect of gel-forming gums on the intestine unstirred layer and sugar transport in vitro .Gastrol., 22: 398-403.

Hoon, K.; Yeun, J.; Won-Hong, W.; Kynng-Soo, J. and Nyeon, J.G. (2001): Effects of the ethanol extract of Cichorium Intybus on the immunatoxicity by ethanol. Immunol., 28(8): 112-119. 
Anna, B.; Ibolya, K.; Krisztina, H.; Eva, A. and Sqnes, K. (2003): Effects of chicory on Pancreas status of rats in experimental dislipidimia J. Ethnopharmcol., 48(1): 141-146.

Katsumata, K. and Katsumata, Y. (1990): The potentiating effect of simultaneous administration of tolbutamide, glibenclamide, and gliclazide on the development of alloxan induced diabetes in rats. Hom. Metab. Res., 22: 51-54.

Kim, M. and Shin, H.K. (1996): The water-soluble extract of chicory reduces glucose uptake from the perfused jejunum in rats. $\mathbf{J}$. Nutri., 126(9): 2236-2242.

Kocsis, I.K.; Hagymàsi, Á.; Kéry, E.; Szôke, and Blàzovics, A.A. (2003): Effects of chicory on pancreas status of rats with experimental dislipidemia.Biologica Szegediensis, 47: 143-146.

Kok, N.; Roberforid, M.; Robet, T. and Delzenne, N. (1996): Involvement of lipogensis in the lower VLDL secretion induced by oligofructose in rats. J.Nutri., 126: $881-890$.

Lairon, D. (1996): Dietary fibres effects on lipid metabolism and mechanisms of action. Europ. J. Clinc. Nutri., 50: 125-133.

Laura, D. and McEntyre, J.R. (2004): Dietary fibres effects on lipid metabolism and the genetic landscape of diabetes. National Library of Medicine, USA.

Lenich, A.C.; Hobanian, A.V.; Brecher, P. and Zannis, V. (1990): Effect of dietary cholesterol and alloxan diabetes on tissue cholesterol and apo lipoprotein E. J. Lipids Res., 32(3): 432-438.

Maechler, P.; Wolheim, C.B.; Bentzen, C.L. and Niesor, E. (1993): Importance of exogenous cholesterol in diabetic rats: effects of treatment with insulin or with an acyl-CoA:cholesterol acyltransferase inhibitor. Ann. Nutr. Metab., 37(4):199-209.

Marcel, B. (1999): Caloric value of inulin and oligofructose. Am. Soc. Nutri. Sci., 129: 1436-1337.

Marie, A.; Christina, R. and Christina, D. (1991): High propionic acid fermentation and mineral accumulation in the cecum of rats adapted to different levels of Inulin.J.Nutri., 121: 1730-1737.

Mc-Letchie, N.G. (2002): Alloxan diabetes: a discovery to induce a minor one. J.Roy. Coll. physic. Eidenburg, 32(2): 134-142.

Mohamed, B.; Hassane, M.; Legssyer, A. and Ziyyat, A. (2002): Medicinal plants in the treatment of diabetes in Morocco. Ethnopharmacol., 10: 33-50.

Niness, K. (1999): Inulin and oligofructose: What are they? Intern. J. Food Sci. Nutri., 129(2): 1402-1406. 
Pushparj, P.N.; Tan, B.K.H. and Tan, C.H. (2001): The hypoglycemic action of the semi-purified fractions of Averrhoa bilimbi in streptozotocin-diabetic rats. Life Sci., 70: 535-547.

Pushparaj, P.N.; Low, J.; Manikandan, B.; Tan, K.H. and Tan, C.H. (2007): Anti-diabetic effects of Cichorium Intybus in streptozotocin-induced diabetic rats. Ethnopharmacol. Singapore, 111: 430-434.

Prince, D.S.; Kamalakkannan, N. and Menon, V.P. (2004): Antidiabetic and antihyperlipidemic effect of alcoholic extract of Syzigium cumin seeds in alloxan- induced diabetic albino rats. J. Ethnopharmacol., 91(203): 209-213.

Rajagopal, K. and Sasikala, K. (2008): Antidiabetic activity of hydroethanolic extracts of Stellata nymphaea flowers in normal and alloxan -induced diabetic rats. Afr. J. Pharmacol., 6: 173-178.

Ranjithakumari, B.D.; Velaytham, P. and Anitha, A. (2007): Comparitive Study on inulin and esculin content of in vitro and vivo plants of cichory (Cichorium Intybus). Intern. J. Pharmaceut. Biologic. Archives, 1: 22-25.

Rao, B.K.; Kesarulu, M.M.; Giri, R. and Apparao, C. (1999): Antidiabetic and hypolipidemic effects of Momordicacymbalaria Hook fruit power in alloxan- diabetic rats. J, Ethnopharmacol., 67(1): 103-109.

Roberfroid, M.B. (2000): Chicory fructo- oligosaccharides and the gastrointestinal tract. J.Nutri., 16(3): 677-679.

Sharma, S.B.; Nasir, A.; Probhu, K.M.; Murthy, P.S. and Dev, G. (2003): Hypoglycemic and hypolipidemic effects of ethanolic extract of seeds of Eugenia jambolana in alloxan - induced diabetic rabbits J. Ethnopharmacol., 85(2-3): 201-206.

Tiedge, M.; Lortz, S.; Drinkgren, j. and Lenzen, S. (1997): Relation between antioxidant enzyme gene expression and antioxidative defense status of insulin producing cells. Diabetes, 46: 1733-1742.

Urias, J.E.; Cani, P.D.; Delmee, E.; Neyrink, A.; Lopesz, M.G. and Delzenne, M.N. (2007): Physiological effects of dietary fructans extracted from Agaves Tequilana and Dasylirion. J.Nutri., 99(1): 254-161.

Wight, W.A. and Niekerk, J.V. (1983): Determination of reducing sugars,sucrose and insulin. J. Agric. Food Chem., 31: 282-285. 
Wilson, G.L.; Patton, N.J.; McCord, J.M.; Mullins, D.W. and Mossman, B.T. (1984): Mechanisms of streptozotocin and alloxan- induced damage in rat beta pancriatic cells. Diabetologies, 27(6): 587-591.

Yassin, M.N.; Ashoush, I.S. and Eldidy, E.M. (2007): Antioxidants content of Chicory leaves extract and its effect as hypolipidemic agent in experimental rats. J. Agric. Sci., 52(3): 177-186.

Yin, X.Z.; Quan, J.S.; Takemichi, K. and Mukoto, T. (2004): Antiatherosclerptic effect soybean isoflavones and soy saponins in diabetic rats. Zhonghua Za Zhi., 35(1): 26-28.

Richmond, N. (1973): Colorimetric determination of total cholesterol and high density lipoproteinlipoprotein cholesterol (HDL-c). Clin. Chem. 19: 1350-1356. 